\title{
Erratum: Effective bilinear-biquadratic model for noncoplanar ordering in itinerant magnets [Phys. Rev. B 95, 224424 (2017)]
}

\author{
Satoru Hayami, Ryo Ozawa, and Yukitoshi Motome
}

(Received 4 July 2018; published 18 July 2018)

DOI: 10.1103/PhysRevB.98.019903

After the publication, we found that the phase boundaries between the triple- $Q n_{\mathrm{sk}}=2$ Skyrmion crystal and other phases in Figs. 9 and 16 were not correct. In Fig. 9, the error is in
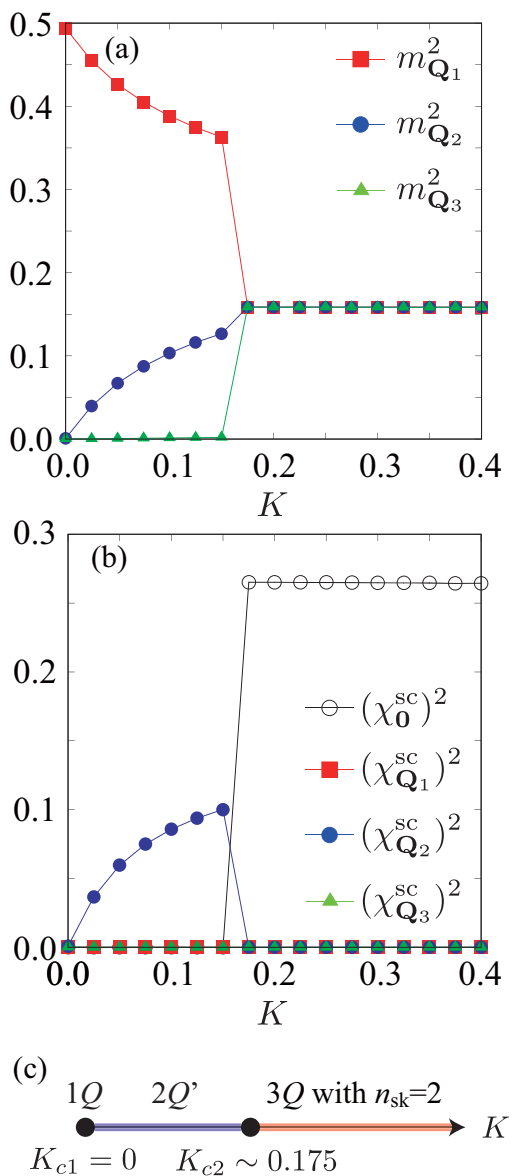

FIG. 9. (a) $K$ dependences of the $\mathbf{Q}_{v}$ components of the squared magnetization obtained by Monte Carlo simulations for the model in Eq. (30). The parameters are $\mathbf{Q}_{1}=(2 \pi / 6,0), \mathbf{Q}_{2}=R(2 \pi / 3) \mathbf{Q}_{1}$, and $\mathbf{Q}_{3}=R(4 \pi / 3) \mathbf{Q}_{1}$. (b) $K$ dependences of the $\mathbf{Q}_{v}$ and uniform $(\mathbf{q}=0)$ components of the squared scalar chirality for the same parameters in (a). (c) Phase diagram for the triangular lattice case. The single- $Q$ helical state $(1 Q)$ is stable only at $K=0$, the double- $Q^{\prime}$ chiral stripe $\left(2 Q^{\prime}\right)$ appears for $0<K \lesssim 0.175$, and the triple- $Q n_{\mathrm{sk}}=2$ Skyrmion crystal ( $3 Q$ with $\left.n_{\text {sk }}=2\right)$ is realized for $K \gtrsim 0.175$.

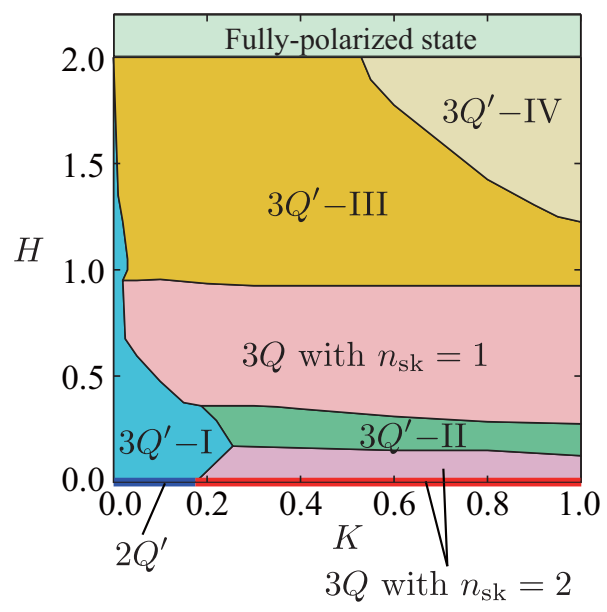

FIG. 16. Phase diagram for the model in Eq. (56) on the triangular lattice with $\mathbf{Q}_{1}=(2 \pi / 6,0), \mathbf{Q}_{2}=R(2 \pi / 3) \mathbf{Q}_{1}$, and $\mathbf{Q}_{3}=$ $R(4 \pi / 3) \mathbf{Q}_{1}$. The spin configuration in each phase is shown in Fig. 17. The phase diagram at $H=0$ coincides with that in Fig. 9(c).

the numerical estimate of $K_{c 2}$ separating the double- $Q^{\prime}$ chiral stripe and the triple- $Q n_{\mathrm{sk}}=2$ Skyrmion crystal: $K_{c 2} \sim 0.37$ in the original paper should be replaced by $K_{c 2} \sim 0.175$. The error is corrected in the revised Fig. 9 presented here. Accordingly, all the values 0.37 in Secs. IV A2, IV B 2, and VIC should read 0.175. Also, the phase diagram in Fig. 16 should be replaced by the revised Fig. 16 presented here. These errors have no effect on our conclusions.

We also found that the value of $\eta \simeq 0.05$ below Eq. (54) was incorrect and should read $\eta \simeq 0.0254$. This leads to a modification of the analytical estimate of $K_{c 2}$. Thus, in Sec. IV B 2, the sentences below Eq. (54) should read "Monte Carlo simulations in Sec. IV A2 give $\eta \simeq 0.0254$ almost independent of $K$. From Eq. (54), we find that the energy of the triple- $Q n_{\text {sk }}=2$ Skyrmion crystal is higher than the single- $Q$ state for small $K$. While increasing $K$, however, the energy of the triple- $Q n_{\mathrm{sk}}=2$ Skyrmion crystal becomes lower for $K \gtrsim 0.145 \tilde{J}$ when we set $\eta=0.0254$. This is qualitatively consistent with the result by numerical simulations in Fig. 9(a), giving the phase boundary at $K_{c 2} \sim 0.175 \tilde{J}$." This error also does not affect our conclusions.

The authors thank Y. Le for pointing out our errors. 